\title{
Surface-defect passivation through complexation with organic molecules leads to enhanced power conversion efficiency and long term stability of perovskite photovoltaics
}

\author{
Weixing Song ${ }^{1}$ and Guozhong $\mathrm{Cao}^{2 *}$
}

Organic-inorganic hybrid lead halide perovskites (e.g., $\left.\mathrm{CH}_{3} \mathrm{NH}_{3} \mathrm{PbX}_{3}, \mathrm{X}=\mathrm{Cl}, \mathrm{Br}, \mathrm{I}\right)$ possess a unique combination of excellent electronic and photoelectrochemical properties including suitable and tunable bandgap, low exciton binding energy in the range of 9-80 $\mathrm{meV}$, high extinction coefficient, and long electron and hole diffusion lengths [1], which make them excellent photovoltaic materials. The absorption coefficient of $\mathrm{CH}_{3} \mathrm{NH}_{3} \mathrm{PbI}_{3}$ is $5.7 \times 10^{4} \mathrm{~cm}^{-1}$ at $600 \mathrm{~nm}$, which is more than one order of magnitude larger than that of silicon. With large absorption coefficient, efficient light harvesting can be obtained with a thin absorber that in turn enhances the open circuit voltage. Photovoltaic solar cells with hybrid lead halide perovskites as active layers have achieved high power conversion efficiencies (PCE) [2], comparable to that of crystalline silicon and thin film solar cells.

The perovskite layer is at the core of perovskite solar cells (PSCs), whose quality would directly determine the device performance. According to the in-situ X-ray scattering studies, the nucleation and crystallization activation energy of the perovskite $\left(56.6-97.3 \mathrm{~kJ} \mathrm{~mol}^{-1}\right)$ is much lower than that of amorphous silicon $\left(280-470 \mathrm{~kJ} \mathrm{~mol}^{-1}\right)$ [3]. The low crystallization energy barrier allows the perovskite films being readily prepared by a variety of low-temperature large-scale fabrication processes including ink-jet printing, doctor-blading and roll-to-roll print. However, such low crystallization energy also permits the easy formation of defects in perovskite crystals, which in turn compromises the material properties and device performance.

The deficiency of long-term stability of the hybrid perovskite material has been one of the greatest barriers to the commercialization of PSCs. The hybrid perovskites are ionic crystals, and the organic and inorganic components are bonded by hydrogen bonds or van der Waals forces. They readily and sensitively respond to even subtle changes in ambient environment, for example, humidity, temperature, oxygen content, and UV light [4]. Phase transition occurs easily and they are easy to decompose into inorganic components due to the loss of organic components with the external stimulus [4]. A great number of approaches have been explored to improve the long-term stability of hybrid perovskite materials. Tuning the perovskite component, constructing two-dimensional perovskite, post treatment, surface passivation, encapsulation and device architecture modification all are investigated as effective strategies to protect the perovskite structure from inside and outside [5].

One of the most important strategies to achieve stable solar cells is to improve the intrinsic stability of the materials. Considering the volatility and thermal instability of methylammonium $\left(\mathrm{CH}_{3} \mathrm{NH}_{3}\right)$ cation, replacing the volatile $\mathrm{CH}_{3} \mathrm{NH}_{3}$ cation with larger and heavier formamidinium (FA, $\mathrm{NHCHNH}_{2}$ ) cation is an effective way [6]. The relatively narrow band gap of $\mathrm{FAPbI}_{3}$ allows broader absorption of solar spectrum, which helps to obtain a high device performance. However, $\mathrm{FAPbI}_{3}$ forms a non-perovskite yellow film consisting of hexagonal phase at room temperature due to the large size of FA cation. Combination of the larger FA cation with the smaller $\mathrm{Cs}$ cation on the $\mathrm{A}$ site to form $\mathrm{FA}_{1-y} \mathrm{Cs}_{y} \mathrm{~Pb}$ $\left(\mathrm{I}_{1-x} \mathrm{Br}_{x}\right)_{3}$ can tune the tolerance factor close to 1 , allowing the formation of the desired black perovskite phase. $\mathrm{FA}_{1-y} \mathrm{Cs}_{y} \mathrm{~Pb}\left(\mathrm{I}_{1-x} \mathrm{Br}_{x}\right)_{3}$ perovskites exhibit enhanced phase

\footnotetext{
${ }^{1}$ Department of Chemistry, Capital Normal University, Beijing 100048, China

${ }^{2}$ Department of Materials Science and Engineering, University of Washington, Seattle WA 98195, USA

* Corresponding author (email: gzcao@uw.edu)
} 


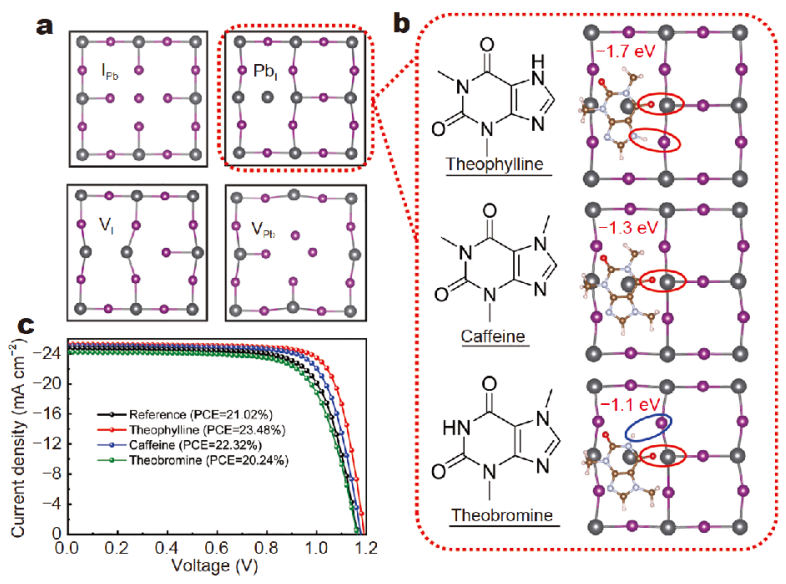

Figure 1 Surface-defect identification and constructive configuration of the $\mathrm{C}=\mathrm{O}$ group in three different chemical environments. (a) Top view of the various types of surface defects. (b) Theoretical models of perovskite with molecular surface passivation of $\mathrm{Pb}_{\mathrm{I}}$ antisite with theophylline, caffeine, and theobromine. (c) $J-V$ curves of perovskite solar cells with or without small-molecules treatment under reverse scan direction. Reprinted with permission from Ref. [9]. Copyright 2019, American Association for the Advancement of Science.

stability as well as enhanced thermal stability [7]. Encapsulation, surface passivation, post treatment and device architecture modification are effective measures to protect the perovskite from the external factors that would directly result in the decomposition of perovskite. The device architecture, the selective contact layers and the metal electrode also have great influence on the stability of the PSCs. In spite of all the efforts with significant progress made in the improvement of PSC stability, it remains a great challenge to achieve the commercialization of PSCs [8].

Most recently, Wang et al. [9] reported an in-depth systematic study on molecular defect passivation approaches through the interaction between organic functional groups and defects in the perovskite and demonstrated both enhanced PCE and long term stability. They first calculated the formation energy of most common defects, lead and iodine vacancies and antisites, using density functional theory (DFT), and identified the $\mathrm{Pb}_{\mathrm{I}}$ antisite defects would predominantly form on the surface (shown in Fig. 1a). The research then focused on the interaction between the surface $\mathrm{Pb}_{\mathrm{I}}$ antisite defect and theophylline, caffeine, and theobromine with three different molecular configurations for defect passivation, and calculated their passivation energies (Fig. 1b). In these molecules, the rigid and conjugated structure as well as the dipoles induced by the heteroatoms tends to increase the intermolecular interaction. This renders them nonvolatile in nature, which is key to the investigation of their interactions with defects in perovskite and longterm stability of the devices. When $\mathrm{N}-\mathrm{H}$ and $\mathrm{C}=\mathrm{O}$ were in an optimal configuration in the molecule, hydrogenbond formation between $\mathrm{N}-\mathrm{H}$ and I (iodine) assisted the primary $\mathrm{C}=\mathrm{O}$ binding with the antisite $\mathrm{Pb}_{\mathrm{I}}$ defect to maximize the surface-defect binding with theophylline, resulting in appreciable defect passivation with both enhanced PCE (Fig. 1c) and long term stability. This work has pointed a direction for the further enhancement and improvement of the PSC performance for their eventual commercialization.

Received 3 January 2020; accepted 7 January 2020; published online 13 January 2020

1 Huang J, Yuan Y, Shao Y, et al. Understanding the physical properties of hybrid perovskites for photovoltaic applications. Nat Rev Mater, 2017, 2: 17042

2 Snaith HJ. Present status and future prospects of perovskite photovoltaics. Nat Mater, 2018, 17: 372-376

3 Moore DT, Sai H, Tan KW, et al. Crystallization kinetics of organic-inorganic trihalide perovskites and the role of the lead anion in crystal growth. J Am Chem Soc, 2015, 137: 2350-2358

4 Domanski K, Alharbi EA, Hagfeldt A, et al. Systematic investigation of the impact of operation conditions on the degradation behaviour of perovskite solar cells. Nat Energy, 2018, 3: 61-67

5 Cheacharoen R, Rolston N, Harwood D, et al. Design and understanding of encapsulated perovskite solar cells to withstand temperature cycling. Energy Environ Sci, 2018, 11: 144-150

6 Eperon GE, Stranks SD, Menelaou C, et al. Formamidinium lead trihalide: a broadly tunable perovskite for efficient planar heterojunction solar cells. Energy Environ Sci, 2014, 7: 982

7 Li Z, Yang M, Park JS, et al. Stabilizing perovskite structures by tuning tolerance factor: Formation of formamidinium and cesium lead iodide solid-state alloys. Chem Mater, 2015, 28: 284-292

8 Huang F, Li M, Siffalovic P, et al. From scalable solution fabrication of perovskite films towards commercialization of solar cells. Energy Environ Sci, 2019, 12: 518-549

9 Wang R, Xue JJ, Wang KL, et al. Constructive molecular configurations for surface-defect passivation of perovskite photovoltaics. Science, 2019, 366: 1509-1513 